\title{
Dewatering Research of Sludge with High Moisture Content
}

\author{
Zhang Yifan ${ }^{1,}$, Z Zhu Shuguang $^{2, b}$ Yuan Hongbin ${ }^{3, c}$ \\ ${ }^{1}$ School of Energy and Power Engineering, Nanjing University of Science and Technology,200 \\ Xiaolingwei ,Nanjing ,China 210094 \\ ${ }^{2}$ School of Energy and Power Engineering, Nanjing University of Science and Technology,200 \\ Xiaolingwei ,Nanjing ,China 210094 \\ b zhushuguang@njust.edu.cn
}

\begin{abstract}
Keywords: Sludge treatment, Dewatering, High moisture content, Biomass, Ultrasonic wave. Abstract. Recent years, municipal sludge received more and more attention as a kind of negative by-product of the urbanization. The technology of dewatering plays a critical role in the sludge handling process. However, domestic academic research in the field of dewatering is not sufficient at present .The dewatering of municipal sludge facing the problem of poor dehydration and high energy consumption .In this paper, the test device is designed and built to test the dewatering characteristics of sludge in different complex working conditions .And by imposing different pressure ,temperature ,mass , and different power of ultrasonic wave ,the correct experimental data are gotten. And the dewatering rate and dehydration power for unit mass can be given by analysis and calculation. Finally the optimal condition was selected according to the principle of minimize power consumption.
\end{abstract}

\section{Introduction}

With the improvement of living standards of national life, the municipal sludge increased rapidly as a kind of by-product of economic. The wastewater treatment plants in Chinese cities dispose more than $1.3 \times 10^{6} \mathrm{t}$ sludge. And the figure is increasing at $10 \%$ every year [1].The sludge can not be treated or utilized without the dewatering process. For the resource utilization and green-type emission, it's vital to develop dewatering device.

At present ,the method of dewatering is various. The vacuum filtration dewatering, press filtration dewatering, centrifugal filtration dewatering and stacked spiral filtration dewatering are relatively mature methods[2,3,4] .Each of these methods has its advantages aims at different kind of sludge and different dewatering effects .In this research, the test device is designed and built to test the dewatering characteristics of municipal sludge in different complex working conditions .Moreover, the ultrasonic is applied to enhance the dewatering effect.

\section{Theoretical models for sludge dewatering}

The municipal sludge has the characteristic of complex structure ,high hydrophilicity and high water content .Vesilind distinguished the water in municipal sludge water into free water, interstitial water ,surface water and water of hydration [5].Theoretically, free water and interstitial water can be removed quickly by high pressure. Water of hydration exist in the cells of plants and animals, it would be free from the surface of cells and separate from cells. But the surface water is attached to the surface of colloidal particles, it could be moved out only by mechanical vibration [6].

Darcy's Law . Darcy's Law is the foundation of the theory of dewatering, and it can be written as:

$$
\frac{\Delta p}{L}=\frac{\mu q}{k A}
$$

Where, $\Delta p$ is the pressure difference, $L$ is the thickness of granular bed , $\mu$ is the viscosity of fluid , $q$ is the flow rate of volume, $A$ is the area of cross sectional . 
The thickness of the filter cake and the ratio of permeability would influence the filtration process as resistance .Here the Ruth Equation can be introduced:

$$
\begin{aligned}
& \frac{d V}{A \cdot d \theta}=\frac{d v}{d \theta}=\frac{p}{\mu\left(R_{c}+R_{m}\right)} \\
& R_{c}=\alpha_{a v} \cdot \frac{W}{A}=\alpha_{a v} \cdot w
\end{aligned}
$$

Where, $R_{c}$ and $R_{m}$ are the resistance of filter cake for unit area and the resistance of filter medium for unit area, $\theta$ is the time for filtering, $V$ is the total filtrate volume after $\theta$ seconds, $v$ is the filtrate volume for unit area, $W$ is the total mass of filter cake, $w$ is the mass of filter cake for unit area, $\mu$ is the viscosity of the filtrate, $\alpha_{a v}$ is the Ruth average filter specific resistance.

The filter specific resistance exported by Ruth is proportional to the mass of filter cake for unit area .According to mass balance equation:

$$
\frac{w}{s}=\rho v+w m
$$

The Ruth Equation for filter rate can be deducted into equation (5):

$$
\frac{d V}{A \cdot d \theta}=\frac{d v}{d \theta}=\frac{p}{\mu\left(\alpha_{a v}\left(\frac{W}{A}\right)+R_{m}\right)}=\frac{p}{\mu\left(\alpha_{a v} w+R_{m}\right)}=\frac{\left(p-p_{m}\right)}{\mu \alpha_{a v} w}=\frac{\left(p-p_{m}\right)(1-m s)}{\mu \alpha_{a v} \rho s v}
$$

Where $p_{m}$ is the pressure imposed on the filter media .

Assume that the mass of solid will generate resistance ,change the form of equation (5), and then take a integral :

$$
\frac{\theta}{v}=\frac{\mu \rho s \alpha_{a v}\left(v+2 v_{m}\right)}{[2 p(1-m s)]}=\frac{1}{K}\left(v+2 v_{m}\right)
$$

Calculation of energy consumption for dewatering. With the measurement data of mass ,pressure ,the filtrate volume for each minute ,the initial and final water content ,the power can be calculated:

$$
W=p s\left(h_{i n i}-h_{\text {fin }}\right)
$$

Where $W$ is the consumption of power, $p$ is the working pressure, $s$ is the extrusion area, $h_{\text {ini }}$ is the initial height of sludge, $h_{f i n}$ is the final height of sludge.

\section{The experimental equipment and method.}

The experimental equipment. The municipal sludge is chosen as sample for all of the experiment , and the dewatering experimental platform is established .The whole scheme of the experiment is just shown in Fig. 1.

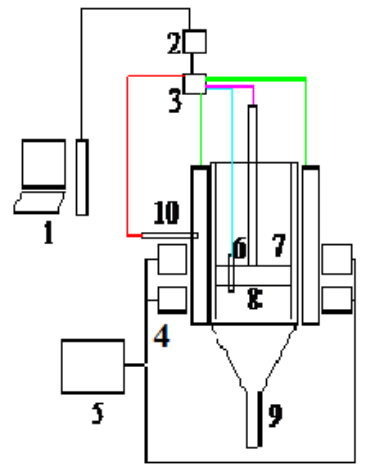

1-computer

2-7520 data acquisition module

3-7018 data acquisition module

4-ultrasonic transducer

5-ultrasonic generator

6-flush membrane pressure sensor

7-piston

8-material cavity

9-filtrate collection device

10-temperature sensor

Fig. 1 The schematic for dewatering test bench.

As the Fig.1 shows that the experimental system is consist of filter press assembly ,ultrasound components and monitoring component. The data of temperature and pressure are collected by the 
7018 data acquisition module .The heated water tank and hand pump are used to adjust the temperature and pressure . ultrasonic transducer is use to produce mechanical vibration for sludge .The frequency of ultrasonic transducer is $28 \mathrm{KHz}$.

The experimental methods. In the experimental device ,the back end of piston is connected with the front end of cylinder. The hand pump is used to provide pressure to squeeze sludge .The water in sludge can through the filter under pressure, the sludge particles stay in the material generator. In this way, the solid and fluid can be separated .

For each experiment, one of the physical variables of temperature, pressure ,mass and the power of ultrasonic wave is changed .And then ,the optimum operating point can be found by comparing with the water content before and after experiment ,the filtrate volume for unit mass and the power consumption for each experiment.

\section{The experiment results}
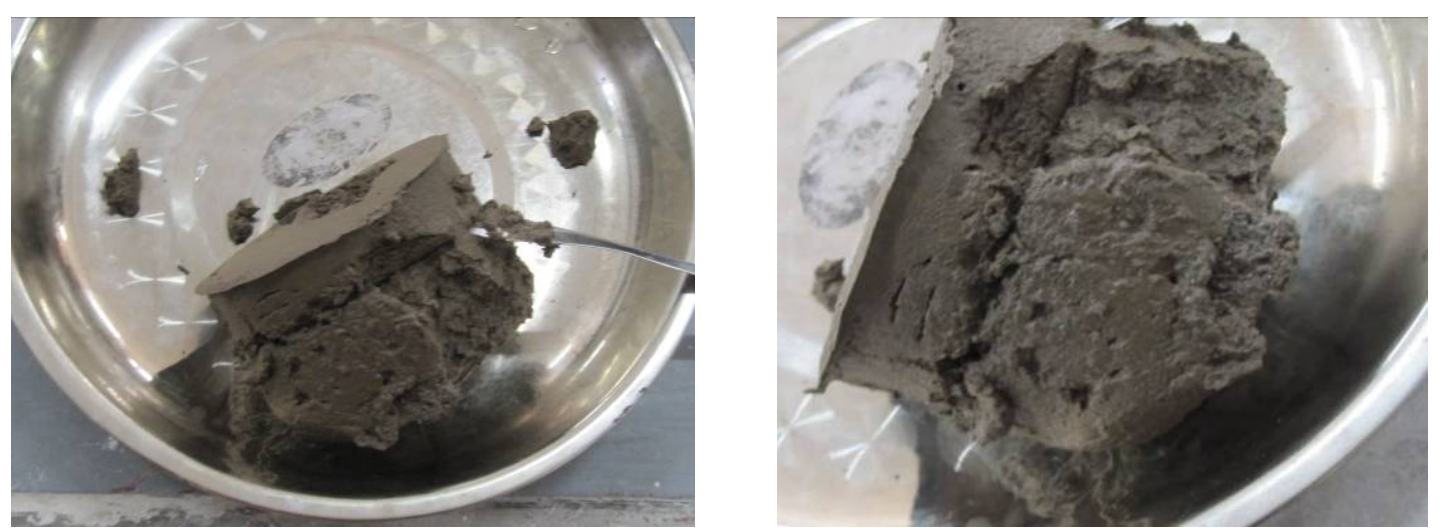

Fig. 2 The samples of municipal sludge after dewatering.

As shown in Fig .2, after filtration, the lower filter cake close to the screen cloth is denser , and the upper cake that far away from screen cloth is looser. This is because the water in the upper portion will encounter more resistance in the filter process, and the filtration effect is poor.

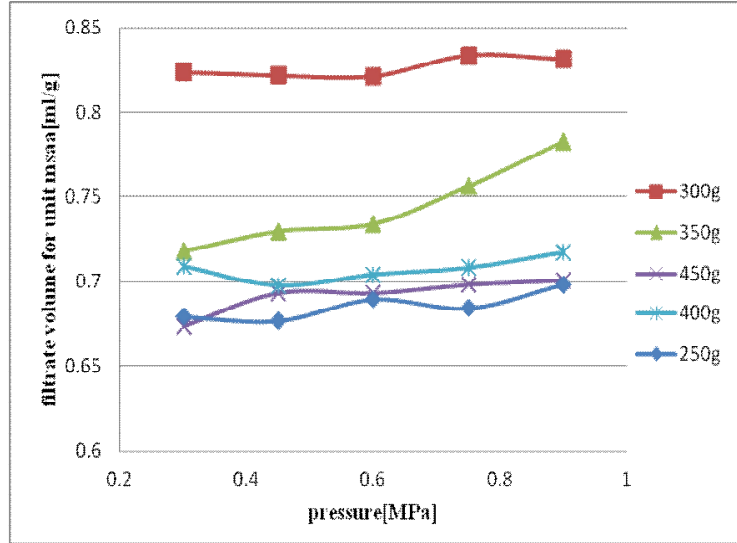

(a)

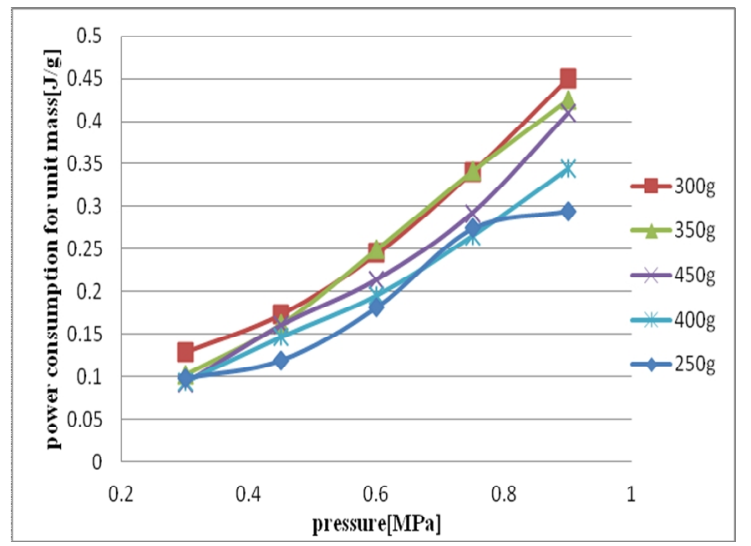

(b)

Fig. 3 a)The realitionship between pressure and feltrate volume for unit mass.

Fig. 3 b)The realitionship between power consumption and feltrate volume for unit mass.

As shown in Fig .3, with the condition of constant pressure, when the mass is not too high, the dewatering rate for unit mass is increasing with the increase of the mass of sludge ,it is because there is a proportion relationship between the filtrate resistance and mass .But when the mass is higher than $350 \mathrm{~g}$, the filtrate volume for unit mass will decrease sharply, this result shows that the 
excessive accumulation of sludge will influence the dewatering effect .It is obvious that with the const mass, the power consumption will increase with the increaing of the pressure.

Tab. 1 The results after varying the temperature.

\begin{tabular}{cccc}
\hline Mass $[\mathrm{g}]$ & $\begin{array}{c}\text { Temperature } \\
{\left[{ }^{\circ} \mathrm{C}\right]}\end{array}$ & Pressure[Mpa] & $\begin{array}{c}\text { The filtrate volume for unit } \\
\text { mass[ml/g] }\end{array}$ \\
\hline 350 & 50 & 0.3 & 0.693571 \\
349.2 & 60 & 0.3 & 0.747136 \\
350.2 & 70 & 0.3 & 0.751571 \\
349.5 & 80 & 0.3 & 0.793419 \\
351 & 30 & 0.3 & 0.673504 \\
\hline
\end{tabular}

As shown in Tab .1, With the condition of constant mass, with the temperature increasing, the dewatering rate for unit mass would increase first and then decrease. This is because that appropriate pressure will provide power for the dewatering process .But if the pressure is much too high, the sludge would be compacting and then block the seepage channel , and finally the dewatering effect will be affected .

Tab.2 The results after varying the power of ultrasonic power

\begin{tabular}{cccc}
\hline mass[g] & Power[W] & Pressure[Mpa] & $\begin{array}{c}\text { The filtrate volume for unit } \\
\text { mass[ml/g] }\end{array}$ \\
\hline 349.7 & 17 & 0.3 & 0.70632 \\
350.1 & 47 & 0.3 & 0.634961 \\
349.7 & 81 & 0.3 & 0.616242 \\
350.1 & 102 & 0.3 & 0.630391 \\
\hline
\end{tabular}

In Tab. 2, it's obvious that the effect of dewatering is better after introducing ultrasonic ,but with the power of ultrasonic increasing, the filtrate volume is decreasing. This is because the mechanical vibration of ultrasonic would destroy the cell structure and separate the water and solid particles .But if the power of ultrasonic power is high, the clearance between solid particle would be small , and the filter specific resistance would increasing. It will against the dewatering process .

\section{Conclusions}

In the experiments ,the unnecessary factors are exclude and the resulting is real and effective ,and the data has very good value for industrial application. In the requirement of water content is less than $20 \%$, according to the principle of minimum power consumption ,the optimum operating point can be found. The physical condition of optimum point is that sludge mass is $350 \mathrm{~g}$, the pressure is $0.3 \mathrm{Mpa}$, the temperature is $30^{\circ} \mathrm{C}$ and the power of ultrasonic wave is $17 \mathrm{w}$.

And further the more, if more different frequencies of ultrasonic waves are put into the experiments ,the dewatering effect influenced by different ultrasonic frequency will be get , and then ,the data will be more rich .

\section{References}

[1] Li Shugeng. Research on Utilization of Dewatered Municipal Sludge to Soil and Its Environmental Effects[D].South China University of Technology,2009.

[2] Huang Zhixin. Study of Structure and Strength of Screw Conveyor and Flow Field in Bowl of Decanter Centrifuge[D]. Beijing University of Chemical Technology ,2007. 
[3] ZHANG Yan-fang ,JIANG Guang-xin. New Type Hot Press Drying-dehyd ration Machine[J].Light Industry Machinery 2009,27(4):86-91.

[4] A.Rushton,A.S.Ward , R.G.Holich. Solid Liquid Filtration and Separation Technology [M].Chemical Industry Press ,2005.

[5] Richard J. Wakeman . Separation technologies for sludge dewatering. [J] .Journal of Hazardous Materials .2007,(144):614 -619.

[6] Akrama Mahmoud . Electrical field:A historical review of its application and contributions in wastewater sludge dewatering[J].Water Research,2010,44:2381-2407. 